\title{
Relato de Caso: Tromboembolismo Pulmonar Maciço em paciente portadora de Trombofilia por deficiência de Proteínas C e S.
}

\author{
Ana Paula Lazaretti ${ }^{1}$; Fernando Covezzi da Silva Filho'; Ludmila de \\ Abreu Castro"; Priscila Marques de Assis ${ }^{1}$; Emanoel Baticini \\ Montanari²; Fábio José Fabrício de Barros Souza ${ }^{3}$. \\ 1.Graduandos em Medicina da Universidade do Extremo Sul Catarinense (UNESC), Criciúma -SC. \\ 2. Graduando em Medicina da Universidade Federal do Rio Grande do Sul (UFRGS). \\ 3. Médico. Especialista em Pneumologia - Santa Casa de Porto Alegre. Mestre em Ciências \\ Pneumológicas pela UFRGS, especialização em Medicina do Sono pela USP e fellowship em \\ Pneumologia e Medicina do Sono na Harvard University. Professor do curso de Medicina - UNESC.
}

\section{Resumo}

Introdução: Tromboembolismo pulmonar é uma doença em que há obstrução da artéria pulmonar ou um dos seus ramos com diminuição do aporte sanguíneo pulmonar para a área irrigada. Um dos fatores de risco são as trombofilias hereditárias. Caso Clínico: G. C. P., 32 anos, sexo femino, do lar. Procurou a emergência por dor torácica em hemitórax esquerdo, de moderada intensidade, ventilatório dependente, súbita. Ao exame físico bom estado geral, lúcida, orientada e consciente, ventilando em ar ambiente e SO2 96\%, eupneica, acianótica, com boa perfusão periférica, afebril. A ausculta cardíaca era normal e na ausculta pulmonar apresentava murmúrios vesiculares diminuídos em base esquerda. Pressão arterial de 95x70mmHg e frequência cardíaca de 85 . Como antecedentes pessoais apresenta cesariana realizada há 45 dias, sem intercorrências, um episódio de trombose venosa profunda em membro inferior direito há 5 anos e tromboembolismo pulmonar há 1 ano, além de 2 episódios prévios de abortos espontâneos. Nega tabagismo. Não há história familiar de 
episódios tromboembólicos. Exames complementares: ECG é sinusal; provas de coagulação normais; gasometria apresentando leve hipoxemia; CPK, CK-MB e Troponina normais; Angiotomografia de Tórax: achados compatíveis com tromboembolismo pulmonar maciço bilateral com hipertensão pulmonar associada a pequenos infartos pulmonares no LID. Exames ambulatoriais anteriores trazidos pela paciente: anticorpos anticardiolipina e anticoagulante lúpico negativos, proteína $\mathrm{C}$ e $\mathrm{S}$ deficientes. Paciente recebeu anticoagulação plena, foi encaminhada a UTI, permaneceu estável hemodinamicamente, devido a isso não necessitou infusão de fibrinolíticos, recebeu alta da UTI após 24 horas, estável clinicamente. Foi iniciado warfarina com alvo de INR entre 2 e 3. Recebeu alta estável e foi encaminhada ao acompanhamento ambulatorial. Discussão: A trombofilia tem por definição uma tendência à trombose decorrente de alterações hereditárias ou adquiridas da coagulação ou da fibrinólise, que levam o individuo a um estado pró-trombótico. Deficiências de Proteína $\mathrm{C}$ e Proteína $\mathrm{S}$, são as que mais cursam com eventos trombóticos e se manifestam como tromboembolismos venosos em indivíduos jovens $(<45$ anos), caráter recorrente e história familiar de eventos trombóticos. Seu acompanhamento e tratamento adequados devem ser estimulados, visto a gravidade dos quadros que podem acometer os pacientes portadores.

Palavras Chave: tromboembolismo pulmonar; trombofilia; proteínas C e S 


\section{Introdução:}

Tromboembolismo pulmonar (TEP) é uma doença cardiopulmonar que ocorre devido a uma obstrução por um trombo formado no sistema venoso profundo, que ao atravessar as câmaras direitas do coração impacta e obstrui a artéria pulmonar ou um dos seus ramos com consequente redução ou cessação do aporte sanguíneo pulmonar para a área irrigada. Nos EUA cursa com uma incidência de 1:1000 indivíduos, sendo que 15\% dos indivíduos morrem nos primeiros 3 meses e cerca de $10 \%$ dos casos o quadro abre com morte súbita. Sua fisiopatologia está relacionada a fatores trombogênicos descritos na Tríade de Virchow (estase do fluxo venoso, lesão ou inflamação endotelial e estados de hipercoagulabilidade), sendo assim qualquer ocasião de imobilização prolongada, cirurgias, traumas, tromboembolismo venoso prévio, doença maligna, obesidade, uso de estrogênio e parto, aumentam possibilidade de um trombo se formar. Ainda como fatores de risco existem as trombofilias, que são desordens de coagulação que criam um estado de hipercoagulabilidade, favorecendo a ocorrência de trombos, dentre elas a deficiência de antitrombina; deficiência de proteínas $\mathrm{C}$ e $\mathrm{S}$; resistência à proteína $\mathrm{C}$ (fator V Leiden); desfibrinogenemia; anticorpo antifosfolipídio/antiocardiolipina; mutação da protrombina; desordens do plasminogênio. A proteína C é uma glicoproteína produzida pelo fígado, células de Leydig e no epidídimo que quando na forma ativa, é capaz de exercer efeito tanto na via intrínseca como extrínseca da coagulação por inativar os fatores Va e VIIIa. Sua deficiência é fator de risco para trombose em cerca de 2 a $5 \%$ dos pacientes e sua herança é do tipo autossômica dominante. Já a proteína $\mathrm{S}$ ativa a proteína $\mathrm{C}$ e inativa os fatores $\mathrm{Va}$ e Xa, muito parecida com a antitrombina III e a proteína $\mathrm{C}$, quando afetado pela deficiência da proteína $\mathrm{S}$ o indivíduo tem até $70 \%$ de risco de vir a ter um episódio de trombose antes dos 60 anos. Ambas proteínas são responsáveis por manter a fluidez sanguínea apesar de fatores coagulantes, porém quando esses fatores se sobrepõem aos mecanismos de controle e regulação acabam favorecendo o aparecimento de eventos trombóticos. Sendo assim, indica-se realizar os testes para trombofilia nos pacientes com menos de 50 anos que apresentam episódios recorrentes de TEP, sem outro fator de risco e em pacientes com história familiar de tromboembolismo venoso, com acometimento de mais de uma geração ou que ocorra em sítios não comuns.

\section{Objetivo:}

Descrever caso de paciente que desenvolveu tromboembolismo pulmonar maciço e foi diagnosticado trombofilia por deficiência de proteínas $\mathrm{C}$ e $\mathrm{S}$.

\section{Metodologia:}


As informações foram obtidas por meio de entrevista com o paciente, revisão do prontuário, registro fotográfico dos exames e revisão da literatura.

\section{Caso Clínico}

G. C. P., 32 anos, sexo femino, casada, natural e procedente de Criciúma-SC, do lar. Procurou o pronto socorro por dor torácica súbita em hemitórax esquerdo, de moderada intensidade, ventilatório dependente, que a acordou durante a madrugada. Refere que há aproximadamente uma semana apresentou inicio de dor leve no mesmo hemitórax, associada a febre de $39^{\circ} \mathrm{C}$, fazendo então uso de assintomáticos e apresentou melhora do quadro no momento. Ao exame físico apresentava bom estado geral, lúcida, orientada e consciente, ventilando em ar ambiente e saturando 96\%, eupneica, acianótica, com boa perfusão periférica. A ausculta cardíaca era normal e na ausculta pulmonar apresentava murmúrios vesiculares diminuídos em base esquerda, sem ruídos adventícios. Estava estável hemodinamicamente com pressão arterial de $95 \times 70 \mathrm{mmHg}$, frequência respiratória de 18 incursões respiratórias por minuto e frequência cardíaca de 85 batimentos por minuto. Paciente apresenta-se em período pós-puerperal, cesariana realizada há 45 dias, sem intercorrências. Não faz uso de nenhuma medicação.

Como antecedentes pessoais apresenta um episódio de trombose venosa profunda em membro inferior direito há 5 anos e tromboembolismo pulmonar há pouco mais de 1 ano, além de 2 episódios prévios de abortos espontâneos. Refere fazer acompanhamento irregular com reumatologista por doença trombofílica, porém não sabe referir qual.

Nega tabagismo ou etilismo.

Não há história familiar de episódios tromboembólicos.

\subsection{Exames complementares:}

ECG de entrada é sinusal, regular, com FC=80bpm. Plaquetas: 173.000, INR: 1.17, TAP: $15.2 \mathrm{seg}$, Atividade de Protrombina: 77\%, KPTT: 159,90. Gasometria de entrada: pH: 7.426, pO2: 68.2, pCO2: 39.7 , HCO3: 25.5, BE:-2.5, SO2: 93.9\%. CPK, CK-MB e Troponina: normais. Angiotomografia de Tórax: Múltiplas falhas de enchimento na artéria pulmonar esquerda e em ramos segmentares para os LLII e LSE, compatíveis com tromboembolismo pulmonar. Tronco da artéria pulmonar medindo $3,2 \mathrm{~cm}$ associado a proeminência das câmaras cardíacas, indicativo de hipertensão pulmonar (figura 1). Pequenas consolidações medindo $1,4 \mathrm{~cm}$ e $1,1 \mathrm{~cm}$ localizados no segmento basal posterior do LID (figura 2), sugestivos de infartos pulmonares. Conclusão: achados compatíveis com tromboembolismo pulmonar maciço bilateral com hipertensão pulmonar associada a pequenos infartos pulmonares no LID. Ecocardiograma transtorácico: Hipertensão arterial pulmonar (estimada pressão pulmonar sistólica máxima em $52 \mathrm{mmHg}$ ), regurgitação mitral leve e sobrecarga de câmaras direitas. 
Traz consigo exames realizados há alguns meses ambulatorialmente: Anticorpos anticardiolipina e anticoagulante lúpico negativos. Proteína C e S deficientes.

\section{2. $\quad$ Evolução}

Após o diagnóstico de tromboembolismo pulmonar maciço confirmado por Angiotomografia de tórax foi instituída anticoagulação plena imediatamente ao diagnóstico com Enoxaparina $1 \mathrm{mg} / \mathrm{kg}$ de 12/12 horas, paciente foi encaminhada para Unidade de Terapia Intensiva (UTI). Devido à estabilidade hemodinâmica, não foi necessária infusão de fibrinolíticos. Na UTI, evoluiu bem, sem instabilidade hemodinâmica permanecendo 24 horas com posterior alta para leito comum. No segundo dia de internação foi iniciado Warfarina, tendo como alvo o RNI entre 2 e 3. Recebeu alta estável hemodinamicamente, sem repercussões sistêmicas e realizará acompanhamento ambulatorial, matendo-a anticoagulada por tempo indeterminado.

\section{Discussão}

A trombofilia tem por definição uma tendência à trombose decorrente de alterações hereditárias ou adquiridas da coagulação ou da fibrinólise, que levam o individuo a um estado pró-trombótico. São classificadas como hereditárias ou adquiridas. A trombofilia hereditária decorre de mutações em fatores envolvidos na coagulação sanguínea, sendo os principais a deficiência das proteínas $\mathrm{C}, \mathrm{S}$ e antitrombina, fator $\mathrm{V}$ de Leiden, mutação G20210A no gene da protrombina (fator II da coagulação). A trombofilia é adquirida quando esta associada a outras condições clínicas, como uso de medicações, neoplasias, síndrome antifosfolípide, imobilização, anticoncepcionais orais, dentre outros fatores.

Deficiências de Proteína $\mathrm{C}$ e Proteína $\mathrm{S}$ apesar de serem menos comuns, são as que mais cursam com eventos trombóticos. Do ponto de vista clínico as trombofilias hereditárias manifestam-se como tromboembolismos venosos características peculiares como: ocorrência em indivíduos jovens ( $<45$ anos), caráter recorrente e história familiar de eventos trombóticos.

Os defeitos trombofílicos podem também causar várias complicações obstétricas, como dificuldade para engravidar, gestações complicadas, abortos de repetição. A análise laboratorial desses distúrbios sempre deve ser investigada.

Alterações no organismo materno durante a gravidez e o puerpério contribuem para o estado de hipercoagubilidade. Associado a isso, a presença de outros fatores de risco para o tromboembolismo requer atenção especial, visto que diagnóstico tardio, tratamento inadequado ou tardio e profilaxia imprópria podem levar à morte materna. A profilaxia não farmacológica recomendada durante a gravidez e no puerpério compreende uso de meias de compressão, hidratação adequada, evitar viagens terrestres e aéreas de longas distâncias e imobilização prolongada. Recomenda-se deambulação 
precoce após o parto e profilaxia mecânica, induzida por exercícios ativos e passivos, incluindo flexão e extensão dos tornozelos, joelhos e quadris, pode evitar a estase venosa; além da possibilidade de anticoagulação farmacológica.

Por fim, deve-se ainda considerar que, por serem defeitos hereditários, a demonstração de um defeito trombofílico congênito determina qual será a investigação dos familiares e aqueles que forem portadores assintomáticos deverão receber orientação adequada em situações de risco, visando evitar a ocorrência de eventos trombóticos.

\section{Tabela e figuras}

Figura1: Tronco da artéria pulmonar medindo $3,2 \mathrm{~cm}$ associado a proeminência das câmaras cardíacas, indicativo de hipertensão pulmonar

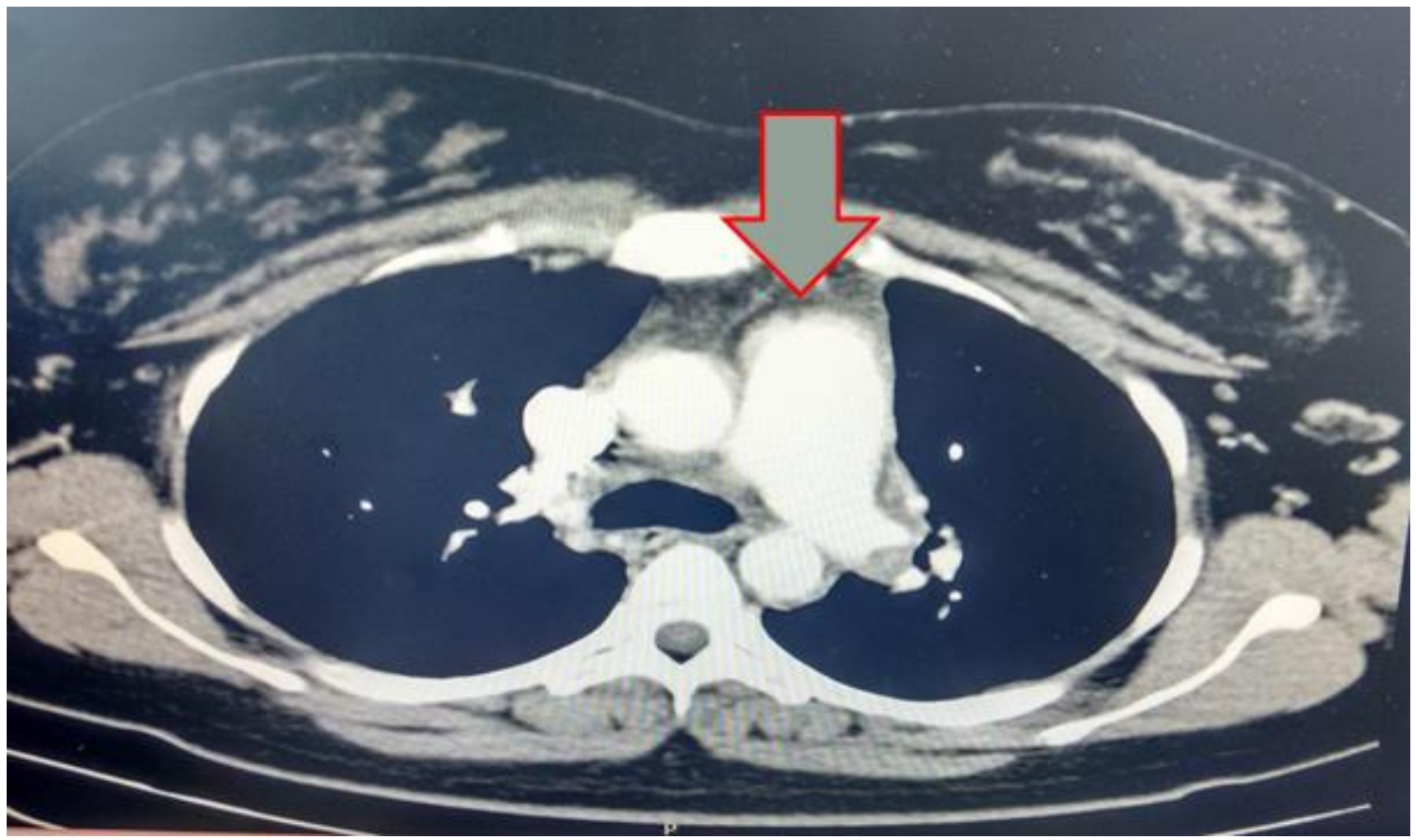

Fonte: arquivo pessoal 
Figura2: consolidações medindo $1,4 \mathrm{~cm}$ e $1,1 \mathrm{~cm}$ localizados no segmento basal posterior do LID

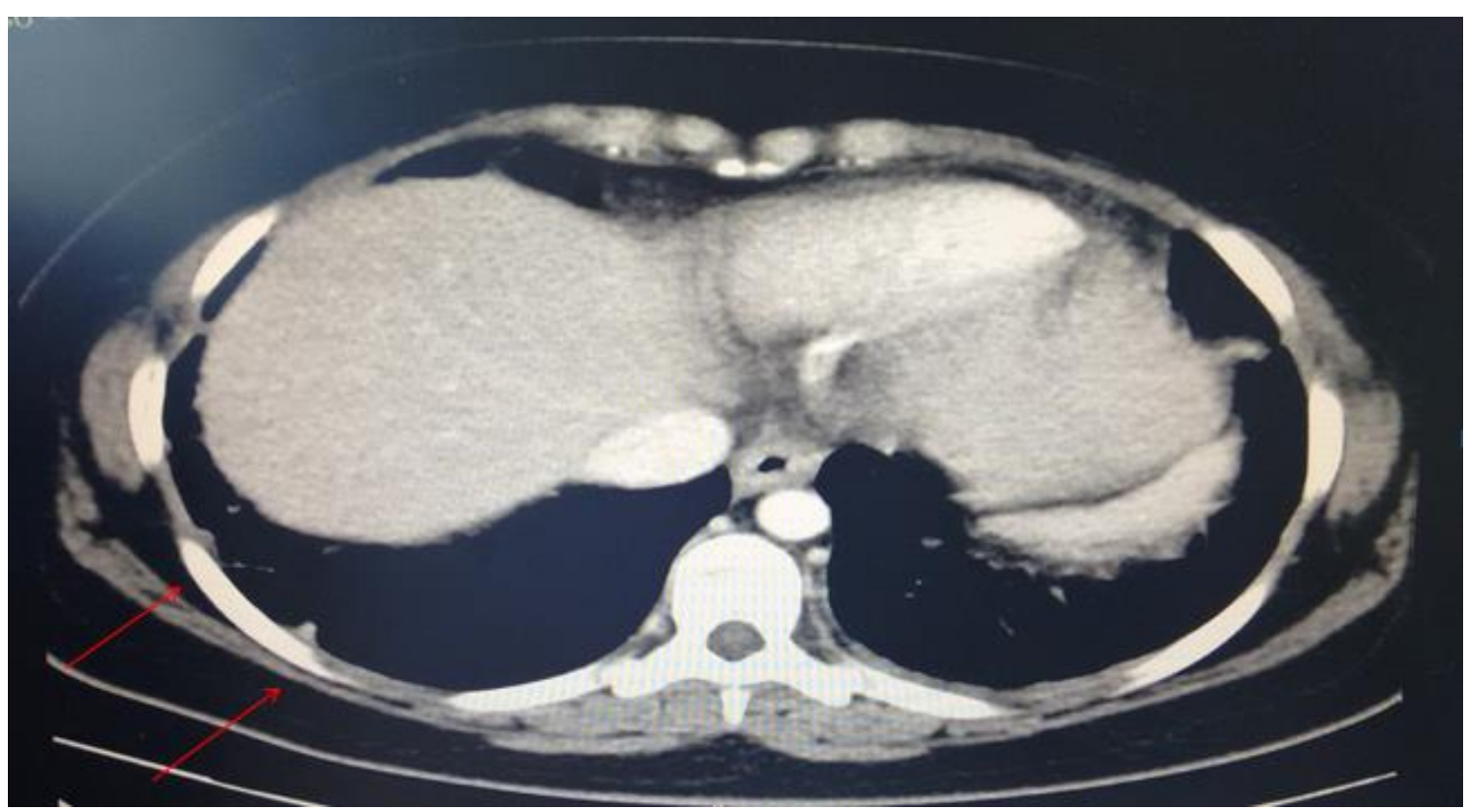

Fonte: arquivo pessoal 


\section{Referências}

Deficiencia de proteínas C y S: marcadores de riesgo trombótico-Revista Cubana Hematología, Inmunología y Hemoterapia. 2013;29 (1):40-47 Lic. Yaneth Zamora-González, Dra. Olga M. Agramonte-Llanes, Lic. Loreta Rodríguez-Pérez

TESE DE MESTRADO. Caracterização molecular da deficiencia de proteína. Luciana Pugliese da Silva. Área de concentração: Farmacologia Titulação: Mestre em Ciencias Médicas.

Trombose venosa profunda: prevalência da deficiência da proteína $\mathrm{S}$ e a interferência da coagulação oral. Rev. Bras. Hematol. Hemoter. vol.25 no.4 São José do Rio Preto 2003.José Maria Pereira de Godoy; Miriam Aparecida Linares; Tais Elizabete Rodrigues; Ronaldo Nardão Mendes; Domingo Marcolino Braile.

Diretriz de Embolia Pulmonar. Arq. Bras. Cardiol. vol.83 suppl.1 São Paulo Aug. 2004.

TROMBOEMBOLISMO PULMONAR: DIAGNÓSTICO E TRATAMENTO. Flávia Alvares; Adriana Ignácio de Pádua; João Terra Filho. Medicina, Ribeirão Preto, 36: 214-240, abr./dez. 2003 
